\title{
The nonlinear analysis of horizontal oil-water two-phase flow in a small diameter pipe ${ }^{1}$
}

\author{
Lu-Sheng Zhai ${ }^{1,2}$, Panagiota Angeli ${ }^{2}$, Ning-De Jin ${ }^{1 *}$, Da-Shi Zhou ${ }^{1}$, Lei Zhu ${ }^{1}$ \\ School of Electrical and Information Engineering, Tianjin University, Tianjin, China ${ }^{1}$ \\ Department of Chemical Engineering, University College London, London, $U K^{2}$
}

\begin{abstract}
Horizontal oil-water two-phase flows are frequently encountered in many industrial processes but the understanding of the dynamic behaviour underlying the different flow patterns is still a challenge. In this study, we first conduct experiments of horizontal oil-water flows in a small diameter pipe, and collect the fluctuation signals from conductance probes. The multiscale power-law correlations of the oil-water flow structures are investigated using Detrended Fluctuation Analysis (DFA) based on the magnitude and sign decomposition of the raw signals. The analysis reveals the scaling behaviour of different flow structures; five conductive flow patterns are indentified based on the magnitude and sign scaling exponents at different time scales. In addition, the transfer entropy (TE) in a state space is used to study the information transferring characteristics of the oil-water mixture flowing past a conductance crosscorrelation velocity probe. The results of TE indicate that the transferring information depends on the flow conditions and can be used to show changes in the flow patterns.
\end{abstract}

Keywords: horizontal oil-water two-phase flow, flow pattern identification, flow structure, magnitude and sign correlations, transfer entropy

\section{Introduction}

Horizontal oil-water two-phase flows are frequently encountered in oil well production and oil transportation. The modelling of the flow parameters in such flows greatly depends on the multi-scale flow structures and the slippage between the two phases. Thus, investigating the

\footnotetext{
* Corresponding author. Tel./fax: +86-22-27407641.

E-mail address: ndjin@tju.edu.cn (N D Jin).
} 
nonlinear dynamic characteristics of oil-water flow structures is of significance for improving the accuracy of measurement and prediction of the flow parameters.

The flow structures forming during oil-water flows can be complex. In early studies they were directly observed via imaging (Russell et al., 1959; Hasson et al., 1970; Arirachakaran et al., 1989). Notably, Trallero et al. $(1995,1997)$ carried out experiments of oil-water flows in a horizontal pipe with an inner diameter of $50.8 \mathrm{~mm}$ and defined six flow patterns, i.e., stratified flow (ST), stratified flow with mixing at interface (ST\&MI), dispersion of water in oil and water flow $(\mathrm{DO} / \mathrm{W} \& \mathrm{~W})$, dispersion of water in oil and oil in water flow (DW/O\&DO/W), dispersion of oil in water flow (DO/W) and dispersion of water in oil flow (DW/O). Angeli and Hewitt $(1998,2000)$ studied oil-water flows in both stainless steel and acrylic test sections using a high frequency impedance probe and indentified a new three-layer pattern. Lovick and Angeli (2004a) grouped all patterns where there are two continuous phases at the top and bottom of the pipe but with drops of one phase into the other to just one pattern named dual continuous (DC) flow. Chakrabarti et al. (2005) observed water-kerosene flow patterns of smooth stratified (SS), stratified wavy (SW), three layer (TL), plug (P) and oil dispersed in water and water (DO/WW), and predicted the pressure drop considering the energy minimization between the two phases. Zhai et al. (2015) from experiments in an acrylic pipe with a small inner diameter of $20 \mathrm{~mm}$ presented a new flow pattern map in which the ST\&MI pattern is subdivided into four groups. In general the oil-water flow patterns are significantly affected by the inlet design, fluid properties, pipe diameter and material, pipe inclination and presence of additives (Brauner, 2003; Rodriguez and Oliemans, 2006; Wegmann and Rudolf von Rohr, 2006; Grassi et al., 2008; Al-Wahaibi et al., 2013; Ibarra et al., 2014; Abubakar et al., 2015). The variety and complexity of the flow structures present great challenges for their analysis and prediction.

In stratified flows the instability of the oil-water interface is associated with the flow pattern transition. Several studies have been carried out to investigate the interfacial features. Chakrabarti et al. (2007) designed a non-intrusive optical probe to investigate oil-water flows, 
and used probability density function analysis and a wavelet multiresolution technique to develop an indicator for stratified flows. Barral and Angeli (2014) measured the wave characteristics of stratified oil-water flows using wire conductance probes and investigated the effects of flow conditions on the power spectrum of the conductance signal. de Castro and Rodriguez (2015) found that the interfacial waves in stratified viscous oil-water flows could be correlated using the Reynolds, Froude and Weber dimensionless numbers. The interfacial wave characteristics can be used in the one-dimensional two-fluid model (Edomwonyi-Otu and Angeli, 2015; Liu et al., 2015; Picchi et al., 2015).

When dispersions occur, the local nonlinear dynamic characteristics are even more complex. Lovick and Angeli (2004b) found that in dual continuous flows droplet concentration and size decreased with increasing distance from the oil-water interface. Kumara et al. (2009, 2010) measured the local phase volume fraction and velocity distribution in a $56 \mathrm{~mm}$ ID steel pipe using a single-bundle gamma density meter and particle image velocimetry (PIV), and found that the degree of mixing between the phases as well as the velocity and turbulence profiles largely depend on the pipe inclination. Morgan et al. (2013) used laser-based optical diagnostic methods to measure droplet size, phase and velocity distributions in stratified and dispersed liquid-liquid flows, and found that the velocity profiles at the lower and upper parts of the pipe correspond to those of laminar flow and turbulent flow respectively. Zhai et al. (2014) developed a parallel wire capacitance probe to measure the cross-correlation velocity of segregated and dispersed oil-water flows in a horizontal pipe and indicated the dependence of the cross-correlation velocity on the flow patterns.

Despite the previous research efforts, the analysis of oil-water flow patterns and their transitions still presents significant challenges. Due to the interplay among many complex factors such as turbulence, changable interfaces, and local relative movement between the phases, horizontal oil-water flows exhibit highly irregular, and unsteady flow structures, which give rise to dissipation, orderly and chaotic patterns. Thus, nonlinear analysis can be beneficial 
for exploring the flow patterns and their transitions.

Nonlinear analysis methods of two-phase flow systems have previously been studied by a number of investigators (Johnsson et al., 2006; van Ommen et al., 2011), particularly focusing on state space analysis (van Ommen et al., 2000; Llauró and Llop, 2006; Cao et al., 2009; Zong et al., 2010; Llop et al., 2012). In a recent paper, we used an Adaptive Optimal Kernel TimeFrequency Representation (AOK TFR) to investigate the flow characteristics of horizontal oilwater flows in terms of total energy and dominant frequency (Zhai et al., 2015). The AOK TFR, however, fails to distinguish the dispersed flow patterns because of their similarities in frequency and total energy.

In this current study, for the characterization of the flow patterns we introduce a multiscale nonlinear analysis method, which is widely used in analysis of physiological signals (Ashkenazy et al., 2001). The long-range correlations of collected conductance signals are investigated by decomposing the signal increment series into magnitude and sign series and extracting their scaling behaviour. Linear and nonlinear properties underlying the oil-water flows are revealed in terms of the magnitude and sign correlations. The observed oil-water flow patterns are identified using a combination of scaling exponents of the magnitude and sign series. In addition, transfer entropy analysis (Schreiber et al., 2000) is used to uncover the flow structure evolution of horizontal oil-water flows based on the conductance signals collected from a cross-correlation velocity probe.

\section{Experiment set up and instrumentation}

The experiments were carried out in the horizontal oil-water flow facility at Tianjin University, shown in Fig. 1. The test fluids used are tap water and No. 15 industry white oil with a viscosity of $11.984 \mathrm{mPa} \cdot \mathrm{s}$. The densities of the oil and water phases are $845 \mathrm{~kg} / \mathrm{m}^{3}$ and $1000 \mathrm{~kg} / \mathrm{m}^{3}$, respectively. The oil-water interfacial tension is $0.035 \mathrm{~N} / \mathrm{m}$. The average temperature at which the experiments were performed was $28^{\circ} \mathrm{C}$. The experiments were carried out by increasing the oil flow rate at a constant water flow rate. The water flow rate was then 
increased to a next value and the procedure was repeated over the entire range of the oil flow rates. The oil, $U_{\mathrm{so}}$, and the water superficial velocity, $U_{\mathrm{sw}}$, varied from 0.106 to $2.579 \mathrm{~m} / \mathrm{s}$ and 0.111 to $2.210 \mathrm{~m} / \mathrm{s}$, respectively. Measurement range and accuracy of each oil flowmeter is $0.005-0.8 \mathrm{~m}^{3} / \mathrm{h}$ with $\pm 0.5 \%$ (volumetric flowmeter), $0.1-2.4 \mathrm{~m}^{3} / \mathrm{h}$ with $\pm 1.5 \%$ (Roots type flowmeter), $2.4-6 \mathrm{~m}^{3} / \mathrm{h}$ with $\pm 1 \%$ (turbine flowmeter), $5-22 \mathrm{~m}^{3} / \mathrm{h}$ with $\pm 0.5 \%$ (turbine flowmeter), and of each water flowmeter is $0.0018-0.1767 \mathrm{~m}^{3} / \mathrm{h}$ with $\pm 0.35 \%$ (electromagnetic flowmeter), $0.1-2.7 \mathrm{~m}^{3} / \mathrm{h}$ with $\pm 0.25 \%$ (electromagnetic flowmeter), $2-9 \mathrm{~m}^{3} / \mathrm{h}$ with $\pm 1 \%$ (Roots type flowmeter), $5-17 \mathrm{~m}^{3} / \mathrm{h}$ with $\pm 0.35 \%$ (vortex flowmeter).

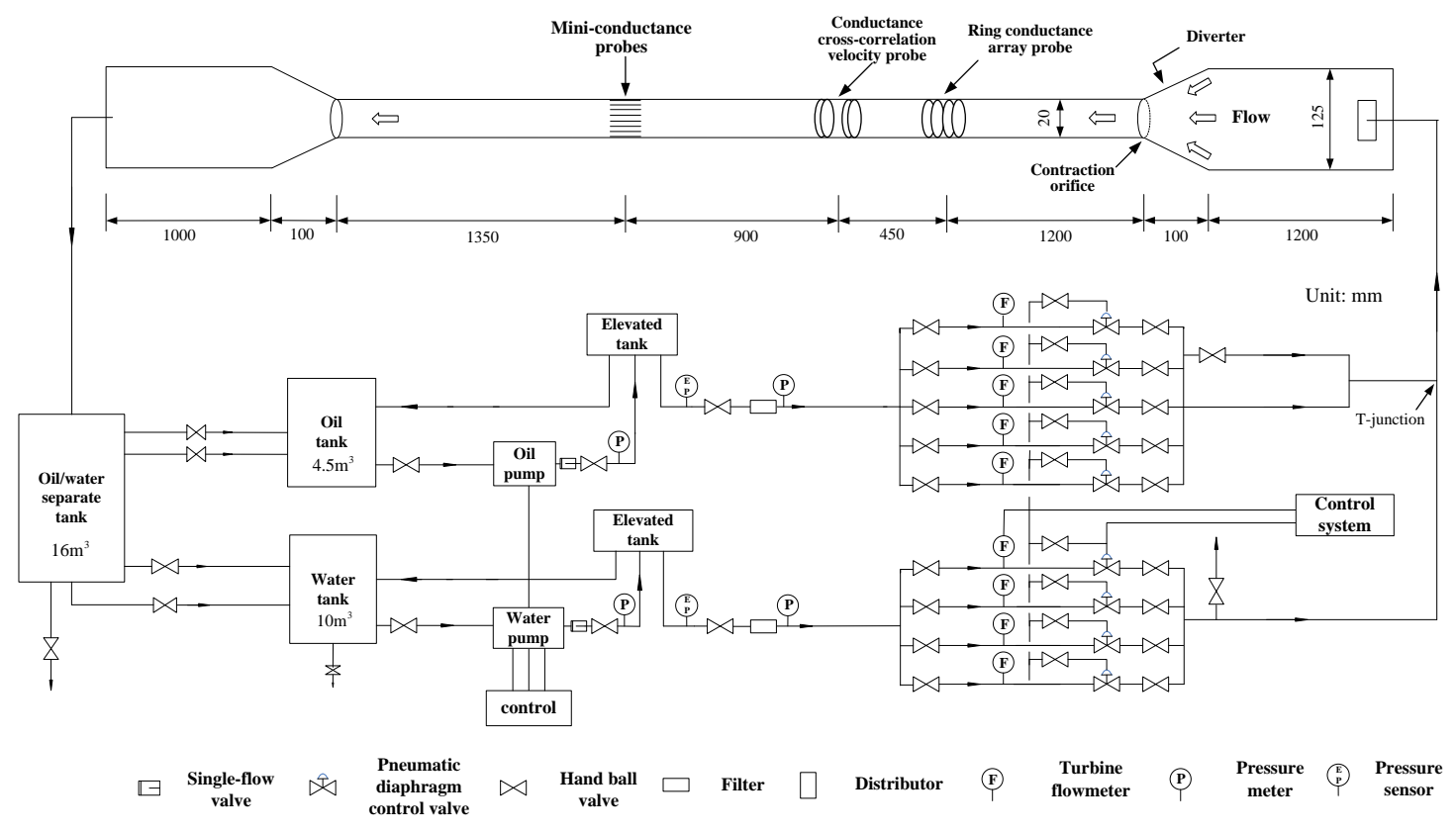

Fig.1. Experimental set up for horizontal oil-water two-phase flows.

For each flow condition, the oil and water phases are pumped from their respective storage tanks and join at a T-junction before entering into an acrylic section with $125 \mathrm{~mm}$ ID and $1.2 \mathrm{~m}$ length. The mixture then enters into the $20 \mathrm{~mm}$ ID acrylic test section via a diverter. The probes mounted on the test section include a ring conductance array probe, a conductance crosscorrelation velocity probe and mini-conductance probes. Detailed descriptions of the probes can be found in Zhai et al. (2015). After the test section, the mixture flows into a separation tank where the two phases are left to separate. Once the separator is full the experiments stop 
and the two phases are returned to their respective storage tanks.

The measurement system of the ring conductance array probe was described in detail in Zhai et al. (2012). The response signals from the ring conductance array probe are collected by channel 0 and 1 of an NI data acquisition card (PXI 4472). The LABVIEW software (version 7.1) is used to configure the parameters of the data acquisition device, display and store the sensor output signals. The signal sampling frequency is set at $2 \mathrm{kHz}$, and the sampling time is 30s. The measurement system of the conductance cross-correlation velocity probe is shown in Fig. 2. The exciting electrodes $\mathrm{E}_{1}$ and $\mathrm{E}_{2}$ are connected with a $20 \mathrm{kHz}$ sinusoidal voltage signal, while the signal from the measuring electrodes is collected with $\mathrm{I} / \mathrm{V}$ converting circuits. The converted voltage signals are processed by a signal conditioning circuit consisting of a phase sensitive demodulator, a low-passing filter and an amplifier. After that the signal conditioning system outputs the upstream signal $V_{\text {m,up }}$ and downstream signal $V_{\mathrm{m}, \mathrm{down}}$ at the same time. The output signals of the conductance cross-correlation probe are collected by channel 2 and 3 of the NI data acquisition card. The signal sampling frequency is set at $6 \mathrm{kHz}$, and the sampling time is $30 \mathrm{~s}$.

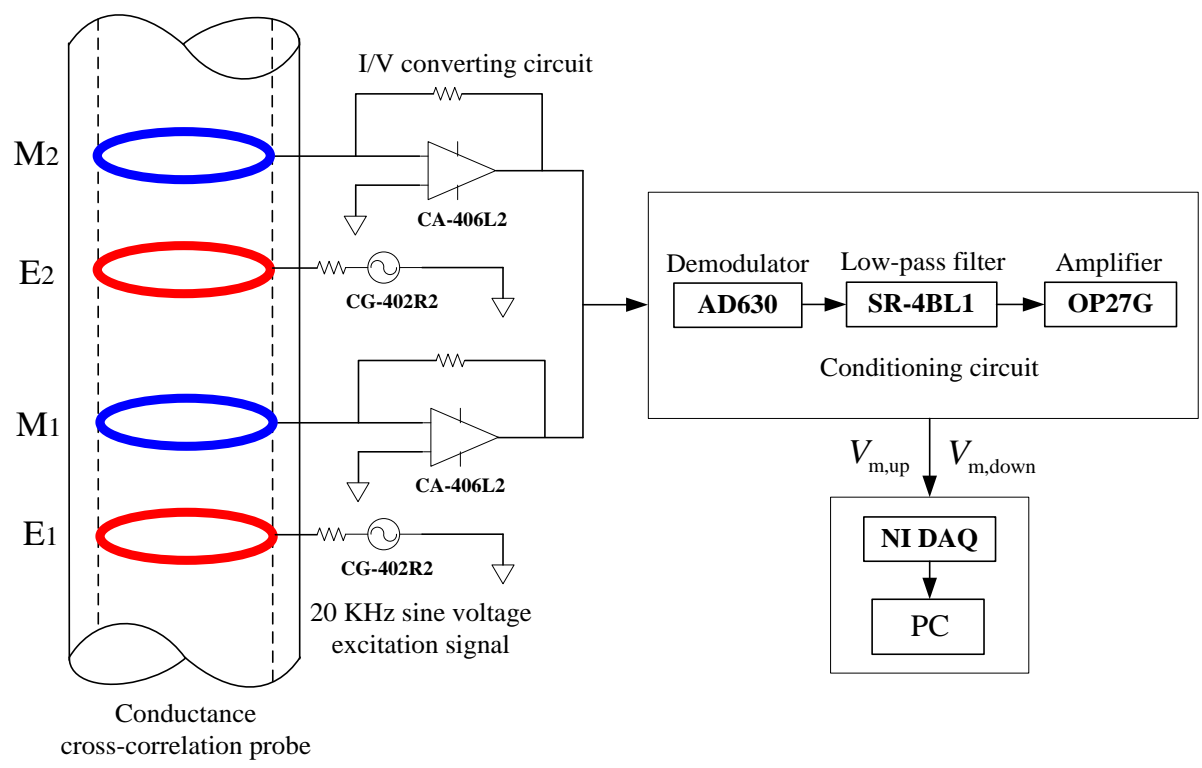

Fig.2. Measurement system of the conductance cross-correlation velocity probe.

The fluctuation signals from the mini-conductance probes are used to indentify the flow patterns, and the respective flow pattern map for the conditions studied is shown in Fig. 3. Six 
flow patterns were observed in agreement with the flow pattern classification proposed by Trallero (1995). As was discussed by Zhai et al. (2015), the ST\&MI is divided into four types. In ST\&MI flow of type I there are a few droplets around the oil-water interface. With further increase of the oil and water flow rates, the number of the oil and water droplets gradually increases, and this type of ST\&MI flow is defined as type II. ST\&MI flow of type III is characterized by an oil-water interface with aggravated and complicated fluctuations at the upper part of the pipe, while the drop entrainment is linked to the wavy interface. In ST\&MI flow of type IV the oil droplets disperse in the continuous water phase at the lower part of the pipe, whereas few water droplets disperse in the continuous oil phase.

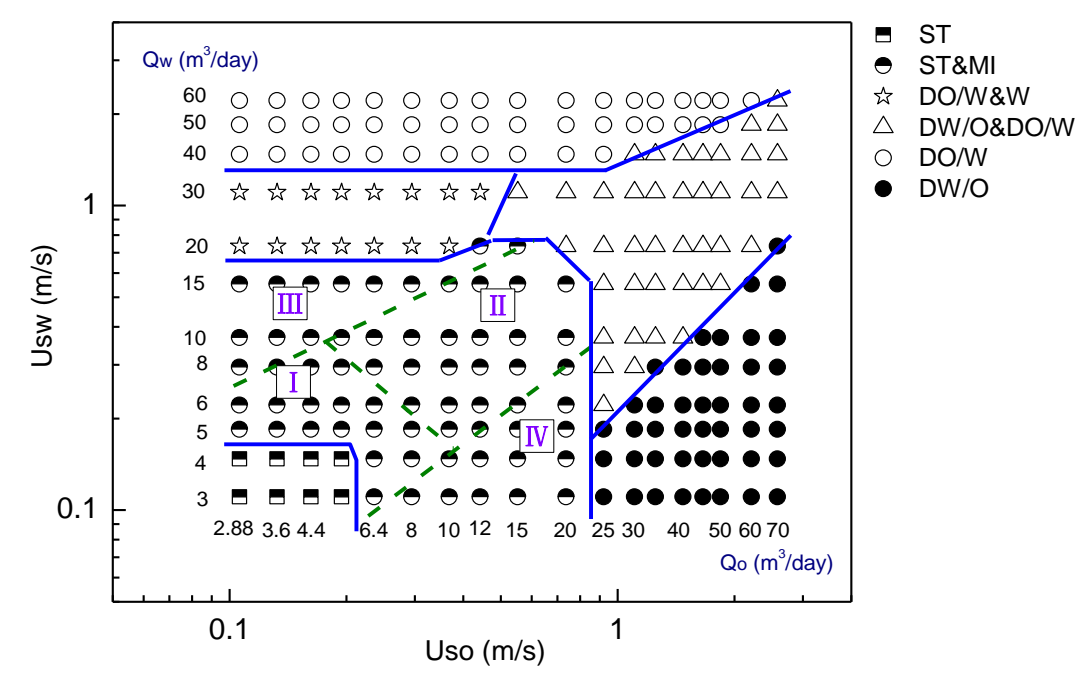

Fig.3. Experimental flow pattern map for the horizontal oil-water two-phase flows (Zhai et al., 2015).

\section{Linear and nonlinear properties of oil-water flow structures}

\subsection{DFA algorithm}

The Detrended Fluctuation Analysis (DFA) allows long-range correlations embedded in a seemingly non-stationary time series to be detected. It also avoids the spurious detection of apparent long-range correlations that are an artefact of non-stationarity (Peng et al., 1995). In the DFA algorithm, a time series $s(i)$ with $N$ data length is firstly integrated as: 


$$
y(k)=\sum_{i=1}^{k}\left(S(i)-S_{a v e}\right)
$$

where $S_{\text {ave }}$ is the average of the time series. The integrated time series is then divided into boxes of equal length $n$. In each box of length $n$, a least-squares line $y_{n}(k)$ is fitted to the data. The integrated time series $y(k)$ is detrended by subtracting the local trend $y_{n}(k)$ in each box. Thus, the root-mean-square fluctuation $F(n)$ is calculated:

$$
F(n)=\sqrt{\frac{1}{N} \sum_{k=1}^{N}\left[y(k)-y_{n}(k)\right]^{2}}
$$

The above computation is repeated over all time scales (box size) to obtain the relationship between $F(n)$ and $n$. If a linear relationship between $F(n)$ and $n$ on a double log graph exists, the fluctuations can be characterized by a scaling exponent $\alpha$, i.e., the slope of the line relating $\log _{F(n)}$ and $\log _{n}$. Values of $\alpha>0.5$ indicate the presence of positive correlation in the time series, $\alpha=0.5$ indicates either the absence of any correlation or the presence of only short-term correlations, and values of $\alpha<0.5$ indicate the presence of an anticorrelation (Peng et al., 1995; Gómez-Extremera et al., 2016).

\subsection{Magnitude and sign DFA}

The output fluctuation signal of a complex dynamic system can be characterized by its magnitude (absolute value) and direction (sign). A given time series from a dynamic system can be decomposed into magnitude and sign series such that their respective scaling properties can be analyzed using DFA (Ashkenazy et al., 2001). In this study, the fluctuation signals from the ring conductance array probe are decomposed into magnitude and sign series, and the DFA algorithm is used to explore the multi-scale properties of the decomposed signals.

In the decomposition of the original signals $S_{S(i)}$ (Ashkenazy et al., 2001), the profile of the increment series is first constructed as $\Delta S(i)=S(i+1)-S(i)$. The increment series is then decomposed into a magnitude series $|\Delta S(i)|$ and a sign series $[\Delta S(i)]$. If $\Delta S(i)>0$, the sign is positive $[\Delta S(i)]=1$, if $\Delta S(i)<0$, the sign is negative $[\Delta S(i)]=-1$, while for $\Delta S(i)=0$, 
$[\Delta S(i)]=0$. Figure 4 shows the signals from the ring conductance array probe under different flow patterns. As can be seen, the signals of ST flows have stable fluctuations, those of DW/O\&DO/W flow are very complex, and the signals of DW/O and DO/W flows have random fluctuations in a very small range. Figure 5 shows the magnitude and sign series decomposed from the ring conductance probe signal obtained during a DW/O\&W flow.

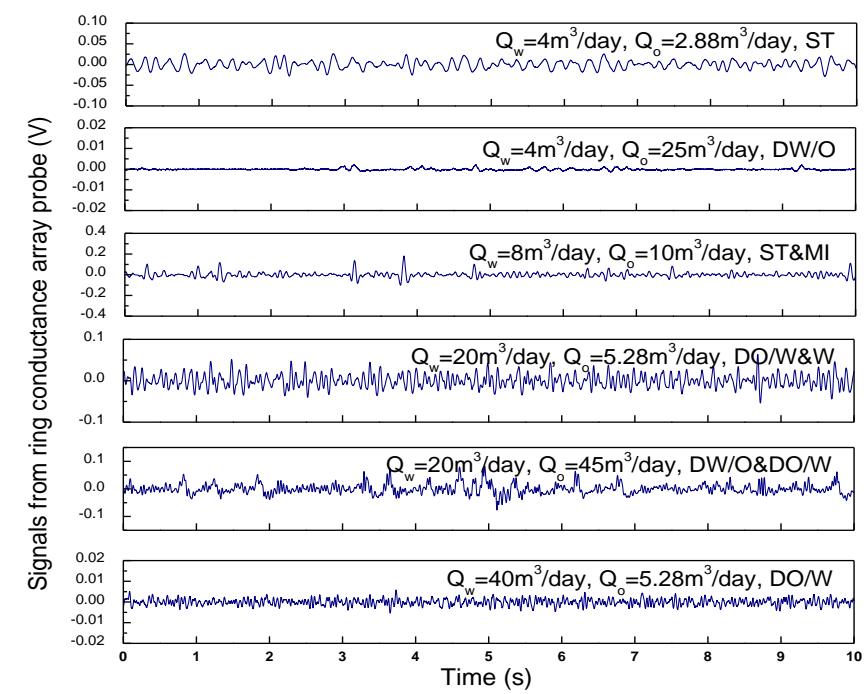

Fig.4. Fluctuation signals from the ring conductance probe under different flow patterns.

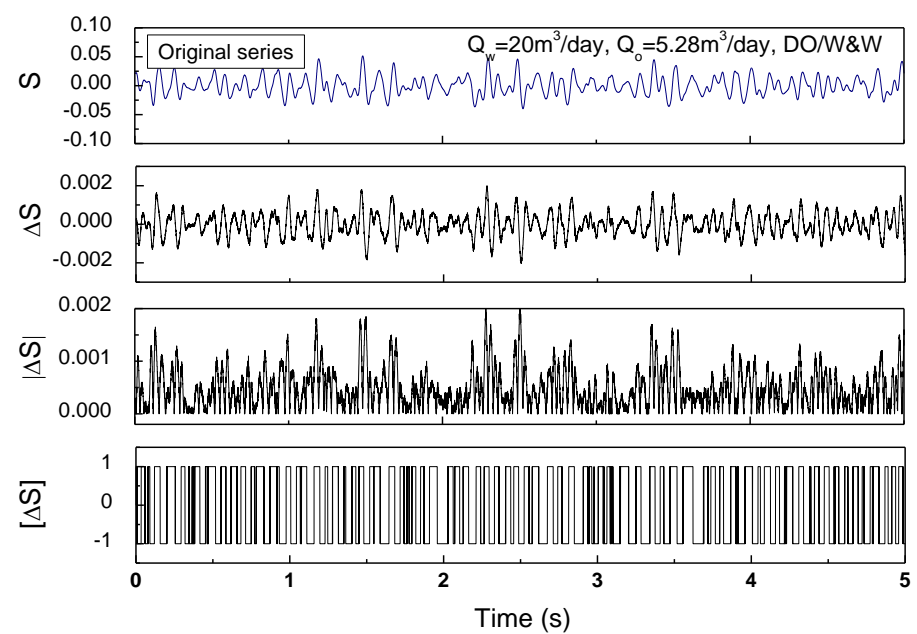

Fig.5. Magnitude and sign series decomposed from the ring conductance probe signal.

Ashkenazy et al. (2001) proposed that the decomposed magnitude series carries the nonlinear properties of the original time series, whilst the sign series relates to the linear properties. Specifically, positive correlations in the magnitude series $\left(\alpha_{\text {mag }}>0.5\right)$ indicate the 
nonlinearity of the original signals. In contrast, linear signals are characterized by an absence of correlation $\left(\alpha_{\mathrm{mag}}=0.5\right)$ in the magnitudes series. The decomposed sign series contains information about underlying dynamics, which is complementary and independent from the original and magnitude series (Shayeganfar, 2012). Positive correlation in the sign series $\left(\alpha_{\text {sign }}>0.5\right)$ indicates that a linear property exists in the original signals, whilst an uncorrelated exponent in sign series $\left(\alpha_{\text {sign }}=0.5\right)$ suggests nonlinear properties.

\subsection{Magnitude and sign correlations of different flow patterns}

In this section we attempt to indentify the different horizontal oil-water flow patterns by investigating the magnitude and sign correlations of the ring conductance array probe signals. The magnitude and sign DFA results of typical flow conditions are shown in Fig. 6 in which DFA-2 (second-order polynomial fitting) is used (Hu et al., 2001; Zhao et al., 2016). As can be seen, the magnitude and sign $F(n)-n$ planes present different scaling behaviour for different flow patterns. Note that, as shown in Fig. 6(a), there is a distinct difference in the magnitude $F(n)-n$ plane of ST and ST\&MI flows (open squares), which probably can be used to indicate flow pattern transitions between stratified and non-stratified flows.

The magnitude scaling exponents at all $(5 \leq n \leq 1000)$ and high $(n \geq 200)$ time scales are respectively represented by $\alpha_{\mathrm{mag}}$ and $\alpha_{\mathrm{mag}}^{\mathrm{H}}$. In Fig. 6(a) the scaling exponents $\alpha_{\mathrm{mag}}^{\mathrm{H}}$ of DO/W flows (open circles) are obviously higher than in the other flow patterns, indicating the strong nonlinearity of DO/W flow at long terms. The sign scaling exponent at high scale $(n \geq 200)$ is represented by $\alpha_{\text {sign }}^{\mathrm{H}}$. As shown in Fig. 6(b), the scaling exponents $\alpha_{\text {sign }}^{\mathrm{H}}$ of ST flows indicate positive correlations $\left(\alpha_{\mathrm{sign}}^{\mathrm{H}}>0.5\right)$, whist the ones of other flow patterns indicate anticorrelations $\left(\alpha_{\text {sign }}^{\mathrm{H}}<0.5\right)$. We conclude that the high values of $\alpha_{\text {sign }}^{\mathrm{H}}$ arise from the linear property of largescale interface waves in ST flow, and could be used to identify stratified flows from conductance probe signals. 


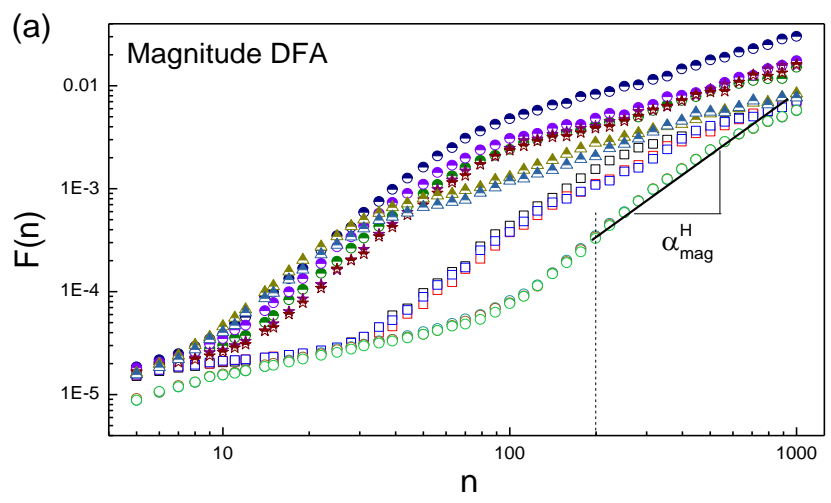

$\mathrm{QW}=4 \mathrm{~m}^{3} /$ day, $\mathrm{Q}=2.88 \mathrm{~m}^{3} /$ day, ST, $\alpha_{\text {mag }}=1.360, \alpha_{\mathrm{mag}}^{\mathrm{H}}=0.868$ $\mathrm{QW}=4 \mathrm{~m}^{3} / \mathrm{day}, \mathrm{Q}=3.6 \mathrm{~m}^{3} / \mathrm{day}, \mathrm{ST}, \alpha_{\mathrm{mag}}=1.278, \alpha_{\mathrm{mag}}^{\mathrm{H}}=1.054$ $\mathrm{QW}=4 \mathrm{~m}^{3} / \mathrm{day}, \mathrm{Q}=4.4 \mathrm{~m}^{3} / \mathrm{day}, \mathrm{ST}, \alpha_{\mathrm{mag}}=1.319, \alpha_{\mathrm{mag}}^{\mathrm{H}}=1.176$ $\mathrm{QW}=4 \mathrm{~m}^{3} /$ day, $Q 0=12 \mathrm{~m}^{3} /$ day, ST\&MI, $\alpha_{\text {mag }}=1.389, \alpha_{\text {mag }}^{\mathrm{H}}=0.794$ $\mathrm{Qw}=4 \mathrm{~m}^{3} /$ day, $\mathrm{Q}=15 \mathrm{~m}^{3} /$ day, ST\&MI, $\alpha_{\mathrm{mag}}=1.509, \alpha_{\mathrm{mag}}^{\mathrm{H}}=0.865$ $\mathrm{QW}=4 \mathrm{~m}^{3} /$ day, $\mathrm{Q}=20 \mathrm{~m}^{3} /$ day, ST\&MI, $\alpha_{\text {mag }}=1.394, \alpha_{\text {mag }}^{\mathrm{H}}=0.820$ $\mathrm{Qw}=20 \mathrm{~m}^{3} / \mathrm{day}, \mathrm{Q}=4.4 \mathrm{~m}^{3} / \mathrm{day}, \mathrm{DO} / \mathrm{W} \& \mathrm{~W}, \alpha_{\mathrm{mag}}=1.444, \alpha_{\mathrm{mag}}^{\mathrm{H}}=0.907$ $\mathrm{QW}=20 \mathrm{~m}^{3} / \mathrm{day}, \mathrm{Qo}=5.28 \mathrm{~m}^{3} / \mathrm{day}, \mathrm{DO} / \mathrm{W} \& \mathrm{~W}, \alpha_{\mathrm{mag}}=1.439, \alpha_{\mathrm{mag}}^{\mathrm{H}}=0.895$ $\mathrm{QW}=40 \mathrm{~m}^{3} / \mathrm{day}, \mathrm{Q}=40 \mathrm{~m}^{3} / \mathrm{day}, \mathrm{DW} / \mathrm{O} \& \mathrm{DO} / \mathrm{W}, \alpha_{\mathrm{mag}}=1.180, \alpha_{\mathrm{mag}}^{\mathrm{H}}=0.707$ $\mathrm{QW}=40 \mathrm{~m}^{3} /$ day, $\mathrm{Q}=45 \mathrm{~m}^{3} / \mathrm{day}, \mathrm{DW} / \mathrm{O} \& \mathrm{DO} / \mathrm{W}, \alpha_{\mathrm{mag}}=1.197, \alpha_{\mathrm{mag}}^{\mathrm{H}}=0.772$ $\mathrm{QW}=60 \mathrm{~m}^{3} /$ day, $\mathrm{Qo}=2.88 \mathrm{~m}^{3} / \mathrm{day}, \mathrm{DO} / \mathrm{W}, \alpha_{\mathrm{mag}}=1.245, \alpha_{\mathrm{mag}}^{\mathrm{H}}=1.692$ $\mathrm{QW}=60 \mathrm{~m}^{3} / \mathrm{day}, \mathrm{Qo}=3.6 \mathrm{~m}^{3} / \mathrm{day}, \mathrm{DO} / \mathrm{W}, \alpha_{\mathrm{mag}}=1.241, \alpha_{\mathrm{mag}}^{\mathrm{H}}=1.699$ $\mathrm{QW}=60 \mathrm{~m}^{3} / \mathrm{day}, \mathrm{Q}=4.4 \mathrm{~m}^{3} / \mathrm{day}, \mathrm{DO} / \mathrm{W}, \alpha_{\text {mag }}=1.246, \alpha_{\text {mag }}^{\mathrm{H}}=1.714$

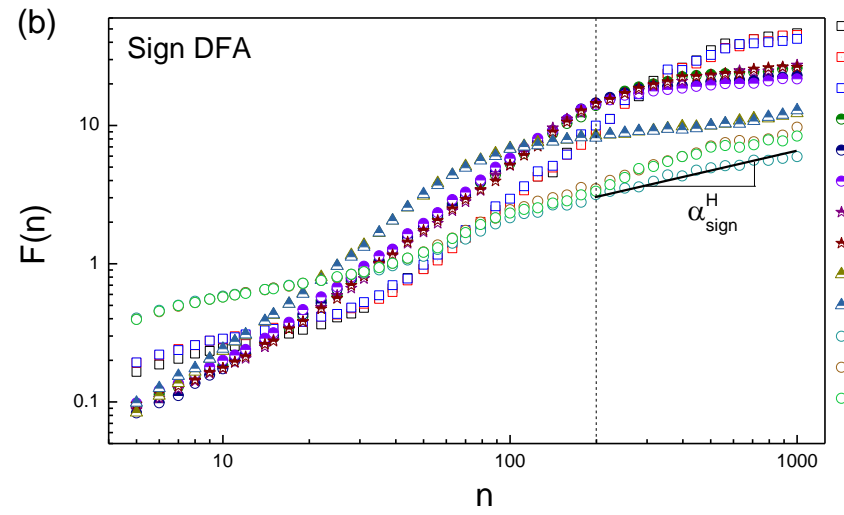

$\mathrm{QW}=4 \mathrm{~m}^{3} / \mathrm{day}, \mathrm{Qo}=2.88 \mathrm{~m}^{3} / \mathrm{day}, \mathrm{ST}, \alpha_{\mathrm{sign}}^{\mathrm{H}}=0.919$ $Q w=4 \mathrm{~m}^{3} / \mathrm{day}, \mathrm{Qo}=3.6 \mathrm{~m}^{3} / \mathrm{day}, \mathrm{ST}, \alpha_{\mathrm{sign}}^{\mathrm{H}}=0.903$ $\mathrm{QW}=4 \mathrm{~m}^{3} /$ day, $\mathrm{Q}=4.4 \mathrm{~m}^{3} /$ day, $\mathrm{ST}, \alpha_{\mathrm{sign}}^{\mathrm{H}}=0.843$

$Q_{W}=4 \mathrm{~m}^{3} / \mathrm{day}, Q_{0}=12 \mathrm{~m}^{3} /$ day, ST\&MI, $\alpha_{\text {sign }}^{H}=0.292$

$Q_{w}=4 \mathrm{~m}^{3} / d a y, Q_{0}=15 \mathrm{~m}^{3} / d a y, S T \& M I, \alpha_{\text {sign }}^{H}=0.210$

$Q W=4 \mathrm{~m}^{3} /$ day, $Q 0=20 \mathrm{~m}^{3} /$ day, ST\&MI, $\alpha_{\text {sign }}^{H}=0.212$

$\mathrm{QW}=20 \mathrm{~m}^{3} / \mathrm{day}, \mathrm{Qo}=4.4 \mathrm{~m}^{3} / \mathrm{day}, \mathrm{DO} / \mathrm{W} \& \mathrm{~W}, \alpha_{\mathrm{sign}}^{\mathrm{H}}=0.347$

$\mathrm{Qw}=20 \mathrm{~m}^{3} /$ day, $\mathrm{Qo}=5.28 \mathrm{~m}^{3} /$ day, DO/W\&W, $\alpha_{\mathrm{sign}}^{\mathrm{H}}=0.335$

$\mathrm{QW}=40 \mathrm{~m}^{3} / \mathrm{day}, \mathrm{Qo}=40 \mathrm{~m}^{3} / \mathrm{day}, \mathrm{DW} / \mathrm{O} \& \mathrm{DO} / \mathrm{W}, \alpha_{\mathrm{sign}}^{\mathrm{H}}=0.230$

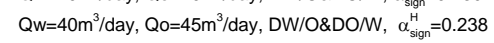

$\mathrm{QW}=60 \mathrm{~m}^{3} /$ day, $\mathrm{Qo}=2.88 \mathrm{~m}^{3} /$ day, DO $/ \mathrm{W}, \alpha_{\mathrm{sign}}^{\mathrm{H}}=0.396$

$\mathrm{QW}=60 \mathrm{~m}^{3} / \mathrm{day}, \mathrm{Q}=3.6 \mathrm{~m}^{3} / \mathrm{day}, \mathrm{DO} / \mathrm{W}, \alpha_{\text {sign }}^{\mathrm{H}}=0.547$

$\mathrm{QW}=60 \mathrm{~m}^{3} / \mathrm{day}, \mathrm{Qo}=4.4 \mathrm{~m}^{3} / \mathrm{day}, \mathrm{DO} / \mathrm{W}, \alpha_{\mathrm{sign}}^{\mathrm{H}}=0.494$

Fig. 6. Results of magnitude and sign DFA under typical flow conditions.

In addition, the scaling exponents $\alpha_{\text {mag }}$ of all DW/O flows are calculated and their average is $1.049 \pm 0.035$ indicating the similarity of DW/O flow with $1 / f$ noise (Peng et al., 1995). This result is in agreement with the nonconductive characteristics of DW/O flows.

Figure 7 shows the combined distribution of magnitude scaling exponent $\alpha_{\text {mag }}$ and sign scaling exponent $\alpha_{\text {sign }}^{\mathrm{H}}$ for the different flow patterns. For DO/W flows the magnitude scaling exponent $\alpha_{\text {mag }}^{\mathrm{H}}$ of is used as a replacement for $\alpha_{\mathrm{mag}}$ in the vertical coordinate. As can be seen, the distribution of $\alpha_{\text {mag }}$ (or $\alpha_{\text {sign }}^{\mathrm{H}}$ ) and $\alpha_{\text {sign }}^{\mathrm{H}}$ in the combined plane can be used to distinguish five flow patterns of horizontal oil-water flows, i.e., ST, ST\&MI, DW/O\&DO/W, DO/W\&W and DO/W flows.

The combined scaling exponents of ST flows appear in zone A of Fig. 7, where the magnitude scaling exponents $\alpha_{\text {mag }}$ change over a small range and the sign scaling exponents $\left(0.7<\alpha_{\text {sign }}^{\mathrm{H}}<1\right)$ show obvious positive correlations. This result is caused by the stable movement 
of the oil-water interface over a long time.

For ST\&MI flows, the combined scaling exponents appear in zone B. As can be seen, the sign scaling exponents distribute in a much larger region, and mainly indicate anticorrelations $\left(\alpha_{\text {sign }}^{\mathrm{H}}<0.5\right)$ of large scale structures in ST\&MI flow. This result agrees with the fact that the entrainment of droplets from the oil-water interface increases the nonlinearity of the large scale structures. In comparison with ST flows, the obvious nonlinearity of the oil-water interface in ST\&MI flow can also be characterized by the larger magnitude scaling exponents $\left(1.3<\alpha_{\text {mag }}<1.6\right)$

For DW/O\&DO/W flows (region C), the complicated droplet distribution and fluctuations of the interface result in weak linear characteristics of the large-scale structures. Thus, the sign scaling exponents $\alpha_{\text {sign }}^{\mathrm{H}}$ change between 0.1 and 0.3 suggesting anticorrelations. Meanwhile, the magnitude scaling exponents $\alpha_{\mathrm{mag}}$ vary between 1 and 1.3, indicating obvious long-range correlations and nonlinear characteristics of this flow pattern.

For DO/W\&W flows, the combined magnitude and sign scaling exponents distribute in zones $\mathrm{B}$ and $\mathrm{C}$, which indicates that a quasi interface exists as suggested in the literature (Hadziabdic and Oliemans, 2007) which makes the DO/W\&W pattern scaling exponents similar to those of ST\&MI and DW/O\&DO/W flows.

For DO/W flows, the magnitude and sign scaling exponents distribute in zone D. The sign scaling exponents $\alpha_{\text {sign }}^{\mathrm{H}}$ suggest anticorrelations $\left(0.2<\alpha_{\text {sign }}^{\mathrm{H}}<0.6\right)$ while the magnitude scaling exponents $\alpha_{\mathrm{mag}}^{\mathrm{H}}$ indicate positive correlations $\left(1.60<\alpha_{\mathrm{mag}}^{\mathrm{H}}<1.91\right)$. This means that DO/W flows show weak linear properties but obvious nonlinearity at long time scales, and this result is significantly dependent on the distribution of the dispersed oil droplets in the continuous water phase. 


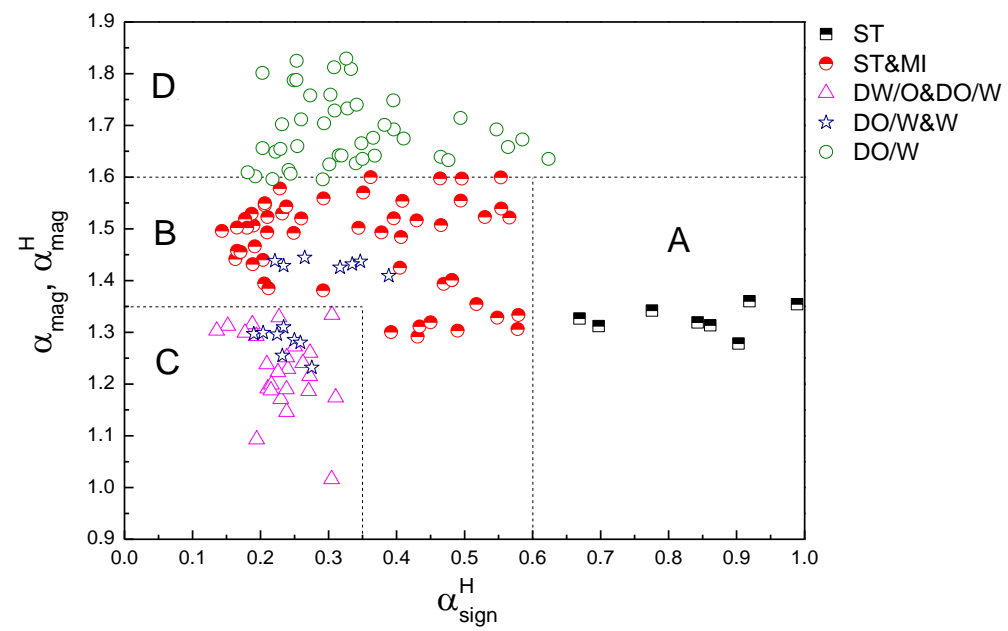

Fig.7. Combined distribution of the magnitude and sign scaling exponents for different flow patterns.

\section{Nonlinear dynamic evolution characteristics of oil-water flow structures}

\subsection{Transfer entropy}

Schreiber et al. (2000) proposed the use of transfer entropy (TE) to quantify the information flow between two systems or between constituent subsystems of a complex system. Recently, the transfer entropy has been used to describe the dynamics and uncover the casual relationship of information transfer in selected complex nonlinear systems, including biological, climate and physical systems (Staniek and Lehnertz, 2008; Wang and Yu, 2012; Ito et al., 2011; Liang and Kleeman, 2005).

In the calculation of transfer entropy (TE) (Schreiber, 2000), the one-dimensional time series $X=[x(1), x(2), \ldots, x(N)]$ with ${ }_{N}$ data length is firstly embedded into a state space with a dimension $d$ and a delay time $\tau$. The reconstructed state space consists of a set of state vectors. Each state vector in the ${ }_{d}$-dimension space is represented by one coordinate point. Hence, the one-dimensional time series form a dynamic track in the state space, in which the $t$ th state vector can be described as

$$
x_{t}^{d}=[x(t), x(t-\tau), x(t-2 \tau), \ldots, x(t-(d-1) \tau)]
$$

where $t$ is a discrete value time-index. Meanwhile, another time series $Y$ is also reconstructed into a state space with the same embedded parameters, and its $t$-th state vector can be given as 


$$
y_{t}^{d}=[y(t), y(t-\tau), y(t-2 \tau), \ldots, y(t-(d-1) \tau)]
$$

The calculation equation of the transferred entropy can be given as

$$
T E(X \rightarrow Y)=\sum_{y_{t+u}, y_{t}^{d_{y}}, x_{t}^{d_{x}}} p\left(y_{t+u}, y_{t}^{d_{y}}, x_{t}^{d_{x}}\right) \log \left(\frac{p\left(y_{t+u} \mid y_{t}^{d_{y}}, x_{t}^{d_{x}}\right)}{p\left(y_{t+u} \mid y_{t}^{d_{y}}\right)}\right)
$$

where $u$ denotes the prediction time, i.e., a discrete value time interval, the arrow denotes the direction of the transferring information, and $p$ denotes the joint probability or the conditional probability.

Equation (5) can be rewritten as a sum of four Shannon entropies according to

$$
T E(X \rightarrow Y)=S\left(y_{t}^{d_{y}}, x_{t}^{d_{x}}\right)-S\left(y_{t+u}, y_{t}^{d_{y}}, x_{t}^{d_{x}}\right)+S\left(y_{t+u}, y_{t}^{d_{y}}\right)-S\left(y_{t}^{d_{y}}\right)
$$

Thus, the problem amounts to computing this combination of different joint and marginal differential entropies. A data efficient approach based on the nearest neighbours techniques and the Kraskove-Stögbauere-Grassberger estimator is used to calculate the Shannon differential entropy (Kraskov et al., 2004; Vicente et al., 2011). The estimator for the transfer entropy can be written as

$$
T E(X \rightarrow Y)=\psi(k)+\left\langle\psi\left(n_{y_{t}^{d y}}+1\right)-\psi\left(n_{y_{t+u} d_{t}^{d y}}+1\right)-\psi\left(n_{y_{t}^{d y} x_{t}^{d d}}\right)\right\rangle t
$$

where $k$ is the number of the nearest neighbour in the nearest-neighbours techniques, $n_{z}$ is the number of the nearest neighbour along the direction of $z, \psi(k)$ is the digamma function, and $\langle\triangleright t \quad$ indicates an averaging over different time points.

For a Lorenz system described by $\dot{x}=-\delta(x-y), \dot{y}=-x z+r x-y, \quad \dot{z}=x y-b z$ (Lorenz, 1963), the TE is calculated between system variables $x, y$ and $z$ with $\delta=10, b=8 / 3$ and initial value $(1,1,1)$. Figure 8 shows the TE values for different system variables against the system parameter $r$ which denotes Rayleigh number. We can see that the TE analysis can effectively indicate the information content and the direction (causality) of the transferring information in a Lorenz system. Specifically, the transferring information between $x$ and $y$ is dependent on the value of $r$ and no remarkable causality is observed between $x$ and $y$. The transferring information from $z$ to $x$ is higher than that from $x$ to $z$, indicating that $z$ is the information source and $x$ is the 
information link, whilst the transferring information from $y$ to $z$ is higher than that from $z$ to $y$, indicating that $y$ is the information source and $z$ is the information link.
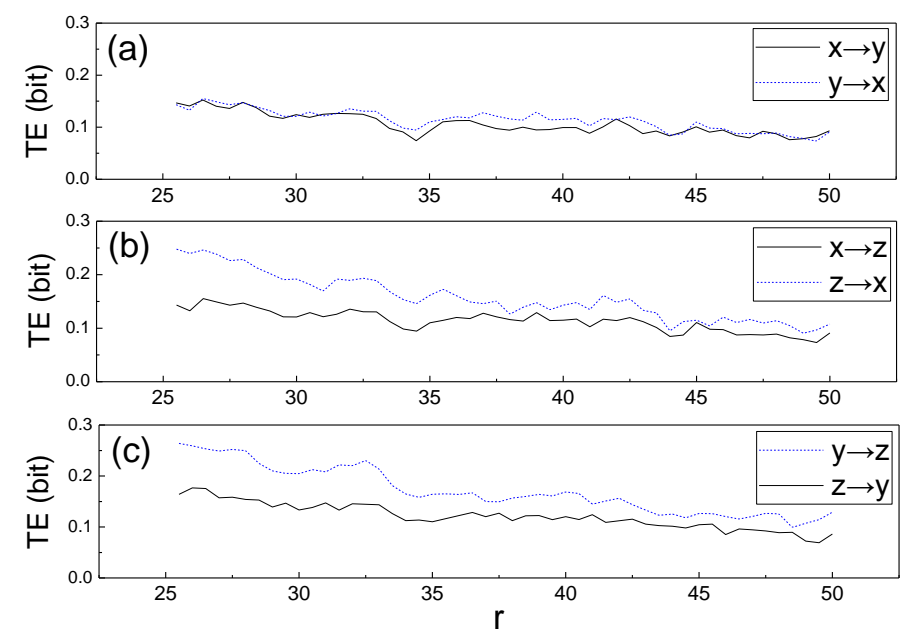

Fig.8. TE between variables of the Lorenz system with the variation of Rayleigh number $r$. The arrows indicate the directions of the transferring information.

\subsection{Transferring information of various oil-water flow structures}

In this section, the TE of oil-water flows is calculated to highlight the nonlinear dynamic evolution characteristics of the various flow structures. In the calculation of the TE for the twophase flows, the upstream electrodes $\mathrm{E}_{1}-\mathrm{M}_{1}$ of the cross-correlation velocity probe are treated as the information source, whilst the downstream electrodes $\mathrm{E}_{2}-\mathrm{M}_{2}$ are the information link. The fluctuation signals from the upstream and downstream electrodes under typical flow conditions are shown in Fig. 9. As can be seen, the upstream and downstream signals have similar fluctuations, while the fluctuations of the downstream signal always follow those of the upstream signal. 

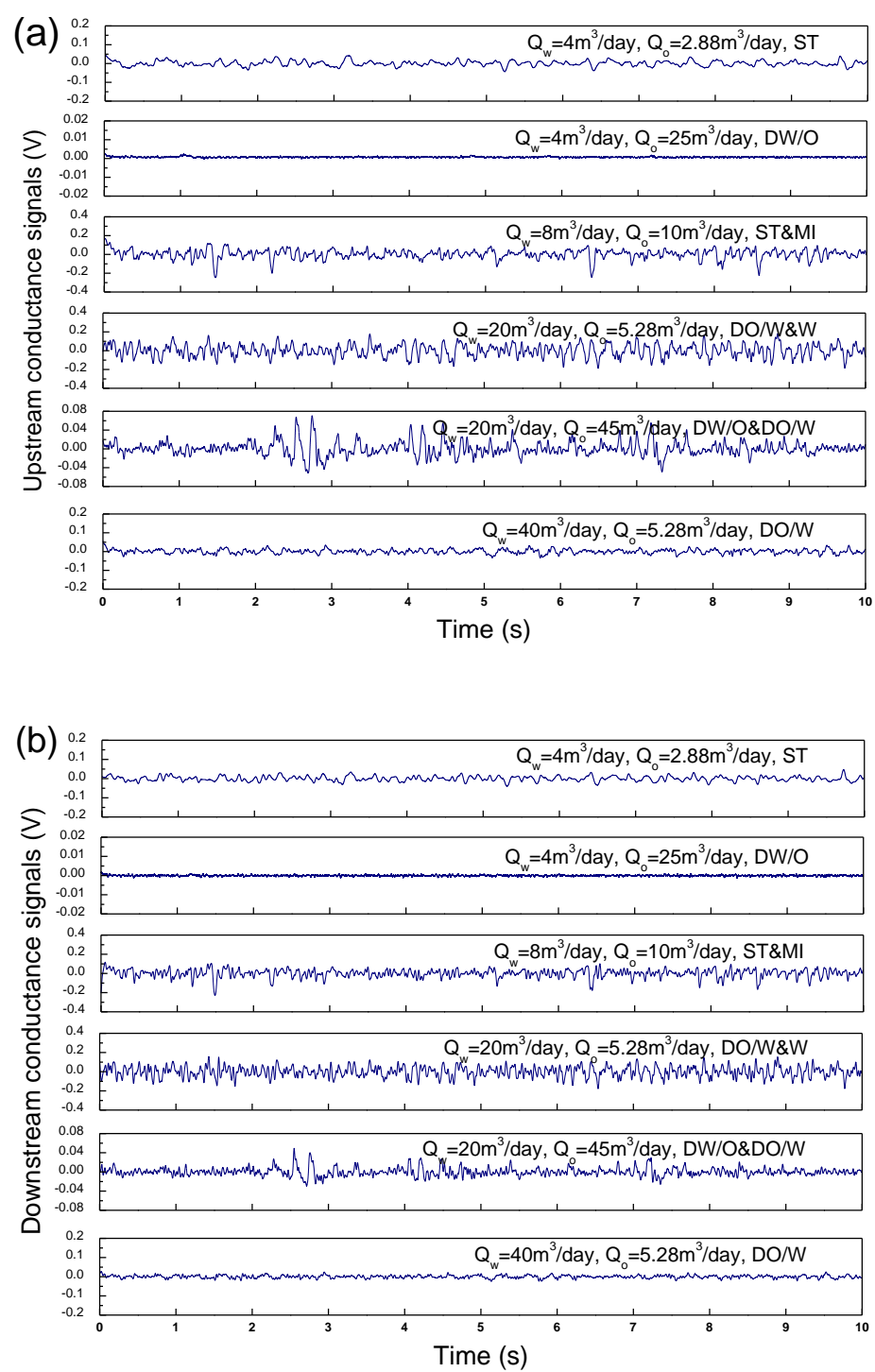

Fig.9. Fluctuation signals from upstream and downstream electrodes of the cross-correlation velocity probe under typical flow conditions.

In the reconstruction of the state space for the one-dimension signals, the embedded dimension $d=3$ and the delay time $\tau_{\tau=2}$ are determined using the method of false nearest neighbours (Abarbanel and Kennel, 1993) and the averaged mutual information method (Liebert and Schuster, 1989), respectively. The values of $u$ and $k$ are both selected as 2 . The calculation results of TE under different flow patterns are shown in Fig. 10.

When the water flow rate is low, as shown in Fig. 3, the flow patterns change from ST, to ST\&MI and then to DW/O flow with the oil flow rate increasing, and accordingly the TE in Fig. 10(a) and 10(b) decreases. Specifically, the TE of ST flow is much higher, which is 
consistent with the fact that ST flow is characterized by a stable interface with large wave lengths, which results in substantial information transferred from upstream to downstream locations. In ST\&MI there is an entrainment of oil and water droplets around the interface which reduces the information transfer to downstream positions. This result indicates that TE could be used to identify the transition from ST flow to ST\&MI flow. With further increase of the oil flow rate, the water phase disperses in the continuous oil phase in DW/O flows. The signals from the upstream and downstream electrodes indicate the characteristics of random noise due to the non-conductive flow. Thus, almost no information transfers from upstream to downstream, and the value of TE is low.

When the water flow rate changes from 5 to $15 \mathrm{~m}^{3} /$ day, as shown in Fig. 3, the flow patterns changes from ST\&MI flow to DO/W\&DO/W flow and then to DW/O flow with increasing oil flow rate. As can be seen in Fig. 10(c) and 10(d), TE gradually reduces with increasing oil flow rate within ST\&MI flows and suddenly increases when the DW/O\&DO/W flow pattern occurs. This result indicates the differences between the segregated and the semi-dispersed flows. These can be compared against the transition criteria such as the one proposed by TorresMonzón (2006) for the boundary between ST\&MI and DW/O\&DO/W flows. Interestingly, in Fig. 10(c), 10(d) and 10(e), the TE of DW/O\&DO/W flow increases first and then decreases. This behaviour may be attributed to the fluctuation characteristics and the location of the oilwater interface. Specifically, when the oil flow rate is not very high, as shown in Fig. 11(a), there exists an unstable layer of oil-in-water and water-in-oil regions between two continuous phases. In this case, the large scale motion of the unstable layer in DW/O\&DO/W dominates the information transmission and results in larger information content transferring from upstream to downstream. With the further increase in the oil flow rate, as shown in Fig. 11(b) and 11(c), the oil-water interface is very close to the bottom of the pipe and the water forms a thin layer, indicating the flow pattern transition from DW/O\&DO/W to DW/O. In this case, the signal fluctuations of DW/O\&DO/W flows are very similar to those of DW/O flows, which 
leads to a decrease of TE.

For DO/W\&W flows, as shown in Fig. 10(e), the TE shows a decreasing tendency as the oil flow rate increases, indicating a similar information transfer characteristics to ST\&MI flows. This result is probably caused by the quasi segregated nature of $\mathrm{DO} / \mathrm{W} \& \mathrm{~W}$ flows and is consistent with the scaling exponent distribution of DO/W\&W flows in Fig. 7.

At high water flow rate, the flow patterns change from DO/W to DW/O\&DO/W with increasing oil flow rate. These patterns are characterised by random motions of the dispersed droplets and have homogeneous flow characteristics. In these patterns it is expected that similar flow structures pass the upstream and downstream electrodes. Accordingly, as shown in Fig. 10(f), the transferring information is high and does not change with increasing oil flow rate.
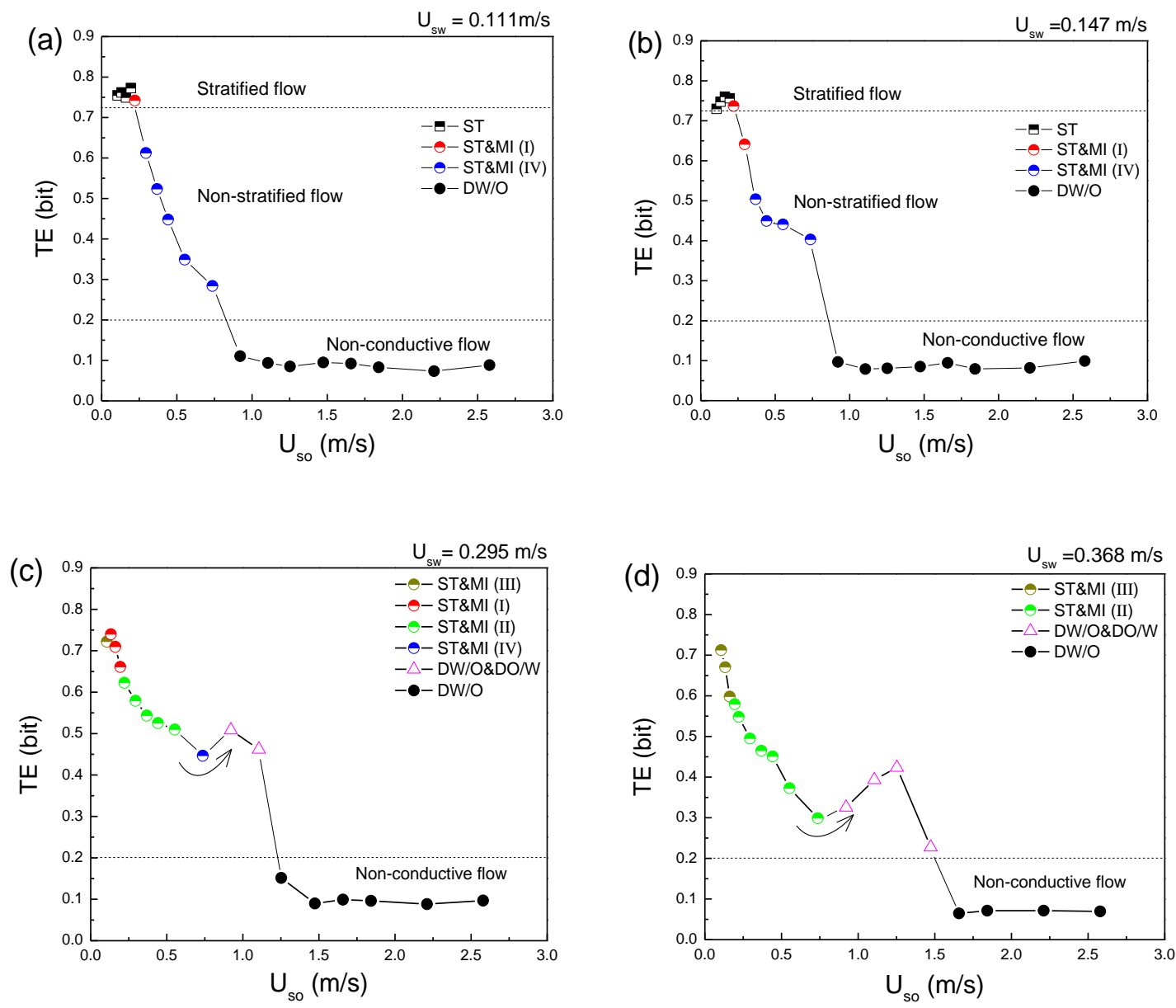

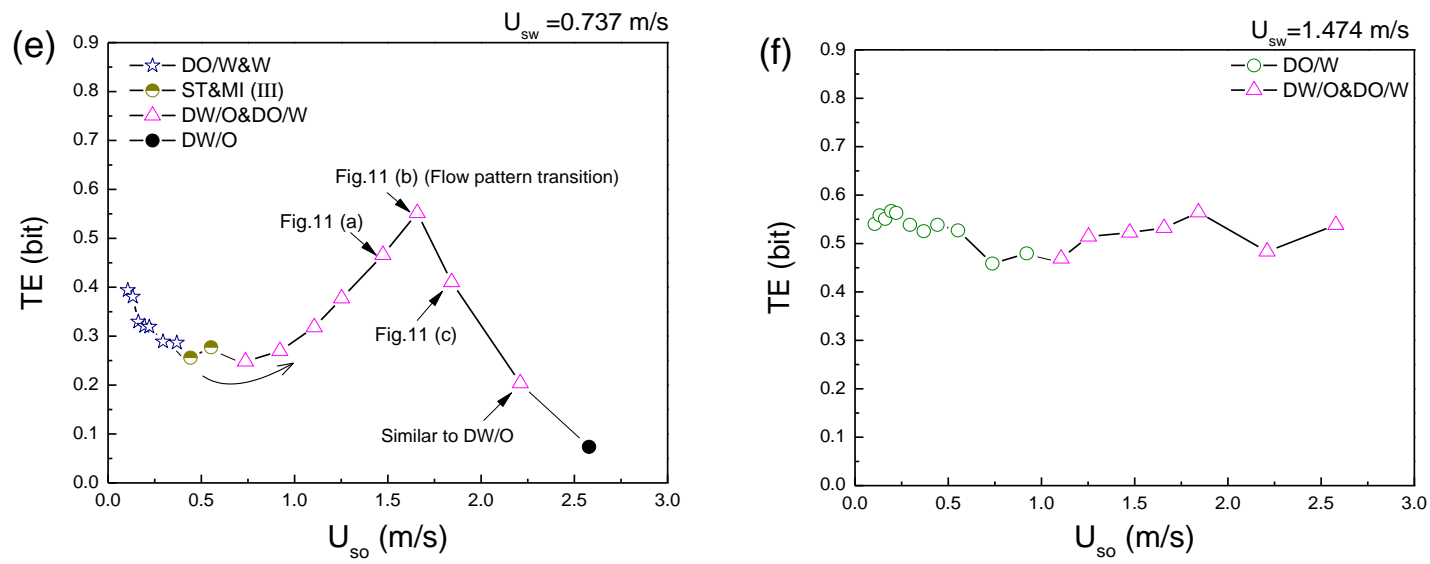

Fig.10. Evolution characteristics of oil-water two-phase flow structures based on transfer entropy.

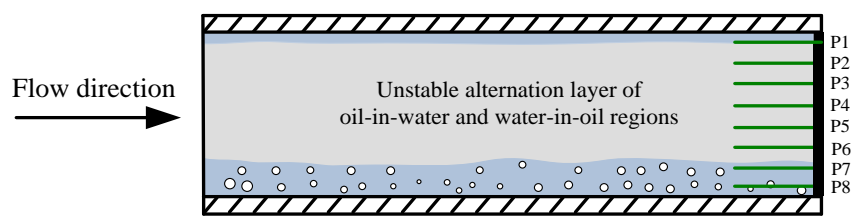

(a) Usw $=0.737 \mathrm{~m} / \mathrm{s}$, Uso $=1.474 \mathrm{~m} / \mathrm{s}, \mathrm{DW} / \mathrm{O} \& \mathrm{DO} / \mathrm{W}$

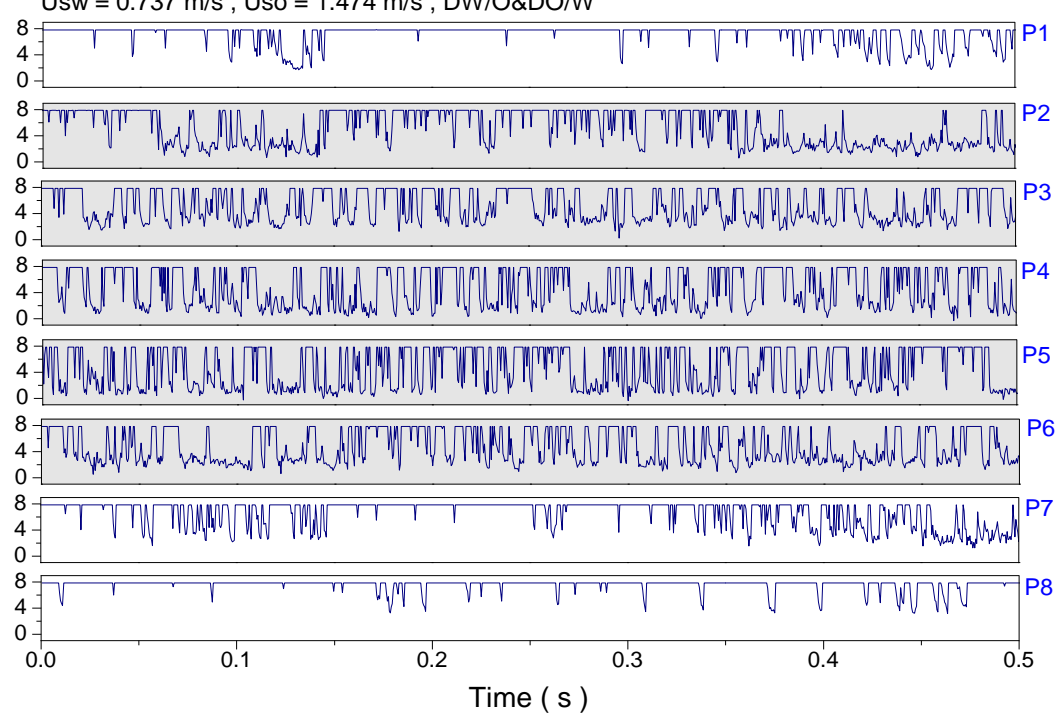





Fig.11. Signals from the mini-conductance probes under typical DW/O\&DO/W flows. The letter P denotes the probes. Eight probes are fixed at equal distances in the pipe along the center line of the pipe cross-section. The low and high voltage levels in the signals represent the appearance of the nonconductive oil phase and the conductive water phase, respectively. (a) The signal fluctuations of probe 3 to probe 6 indicate an unstable 
layer of oil-in-water and water-in-oil regions; (b) (c) Probe 1 to probe 7 are immersed in an water-in-oil region, whilst probe 8 is immersed in an oil-in-water region. Note that probe 3 and probe 4 indicate complex distributions of water droplets in the continuous oil phase.

\section{Conclusions}

There are obvious multi-scale characteristics in the horizontal oil-water two-phase flow structures, such as the interface fluctuations and the motion of the dispersed droplets. In this study, magnitude and sign Detrended Fluctuation Analysis (DFA) is used to uncover the nonlinear characteristics of the multi-scale flow structures. The magnitude and sign scaling exponents at different time scales are combined to distinguish the various flow patterns. The results of magnitude and sign DFA show a considerable potential in characterizing the multiscale dynamic behaviour underlying the complex oil-water two-phase flow systems.

When the oil-water phases flow past a cross-correlation velocity probe, the information of the flow structures can be transferred from the upstream to downstream electrodes. The transfer entropy (TE) is calculated in a state space to indicate the information content of the flow structures transferring from upstream to downstream. The results indicate that TE can effectively reveal the dynamic evolution characteristics of the horizontal oil-water flow structures. It was also found that TE could indicate flow pattern transitions.

The DFA and the TE analysis carried out in this study were based on the fluctuating signals collected from conductance sensors. In future work we plan to use distributed multiple sensors to explore local flow structures and to enhance the application of DFA and TE in the study of the dynamics underlying complex flow systems.

\section{Acknowledgments}

This work was supported by the National Natural Science Foundation of China (Grant Nos. 41504104, 51527805, 11572220), Natural Science Foundation of Tianjin, China (Grant Nos. 14JCQNJC04200), Specialized Research Fund for the Doctoral Program of Higher Education 
(Grant Nos. 20130032120042) and China Scholarship Council (Grant Nos. 201506255066).

\section{References}

Abarbanel, H. D. I., Kennel, M. B., 1993. Local false nearest neighbors and dynamical dimensions from observed chaotic data. Phys. Rev. E 47, 3057-3068.

Abubakar, A., Al-Wahaibi, T., Al-Hashmi, A. R., Al-Wahaibi, Y., Al-Ajmi, A., Eshrati, M., 2015. Influence of drag-reducing polymer on flow patterns, drag reduction and slip velocity ratio of oil-water flow in horizontal pipe. Int. J. Multiphase Flow 73, 1-10.

Al-Wahaibi, T., Al-Wahaibi, Y., Al-Ajmi, A., Yusuf, N., Al-Hashmi, A. R., Olawale, A. S., Mohammed, I. A., 2013. Experimental investigation on the performance of drag reducing polymers through two pipe diameters in horizontal oil-water flows. Exp. Thermal Fluid Sci. 50, 139-146.

Angeli, P., Hewitt, G. F., 1998. Pressure gradient in horizontal liquid-liquid flows. Int. J. Multiphase Flow 24, 1183-1203.

Angeli, P., Hewitt, G. F., 2000. Flow structure in horizontal oil-water flow. Int. J. Multiphase Flow 26, 1117-1140.

Arirachakaran, S., Oglesby, K. D., Malinowsky, M. S., Shoham, O., Brill, J. P., 1989. An analysis of oil-water phenomena in horizontal pipes. SPE Productions Operations Symposium, SPE 18836.

Ashkenazy, Y., Ivanov, P. C., Havlin, S., Peng, C. K., 2001. Magnitude and sign correlation in heartbeat fluctuations. Phys. Rev. Lett. 86(9), 1900-1903.

Barral, A. H., Angeli, P., 2014. Spectral density analysis of the interface in stratified oil-water flows. Int. J. Multiphase Flow 65, 117-126.

Barral, A. H., Ebenezer, A., Angeli, P., 2015. Investigation of interfacial waves at the inlet section in stratified oil-water flow. Exp. Thermal Fluid Sci. 60, 115-122.

Brauner, N., 2003. Liquid-Liquid Two-Phase Flow Systems, in: Bertola, V. (Ed.), Modelling and Experimentation in Two-Phase Flow. Springer Vienna, Vienna, pp. 221-279. 
Cao, Y., Wang, J., He, Y., Liu, W., Yang, Y., 2009. Agglomeration detection based on attractor comparison in horizontal stirred bed reactors by acoustic emission sensors. AIChE Journal $55,3099-3108$.

Chakrabarti, D.P., Das, G., Das, P.K., 2007. Identification of stratified liquid-liquid flow through horizontal pipes by a non-intrusive optical probe. Chem. Eng. Sci. 62, 1861-1876.

Chakrabarti, D.P., Das, G., Ray, S., 2005. Pressure Drop in Liquid-liquid Two Phase Horizontal Flow: Experiment and Prediction. Chem. Eng. Technol. 28, 1003-1009.

de Castro, M. S., Rodriguez, O. M. H., 2015. Interfacial waves in stratified viscous oil-water flow. Exp. Thermal Fluid Sci. 62, 85-98.

Edomwonyi-Otu, L. C., Angeli, P., 2015. Pressure drop and holdup predictions in horizontal oil-water flows for curved and wavy interfaces. Chem. Eng. Res. Des. 93, 55-65.

Gómez-Extremera, M., Carpena, P., Ch. Ivanov, P., Bernaola-Galván, P. A., 2016. Magnitude and sign of long-range correlated time series: Decomposition and surrogate signal generation. Phys. Rev. E 93, 042201.

Grassi, B., Strazza, D., Poesio, P., 2008. Experimental validation of theoretical models in twophase high-viscosity ratio liquid-liquid flows in horizontal and slightly inclined pipes. Int. J. Multiphase Flow 34, 950-965.

Hadziabdic, M., Oliemans, R. V. A., 2007. Parametric study of a model for determining the liquid flow-rates from the pressure drop and water holdup in oil-water flows. Int. J. Multiphase Flow 33, 1365-1394.

Hasson, D., Mann, U., Nir, A., 1970. Annular flow of two immiscible liquids. Can. J. Chem. Eng. 48, 514-520.

Hu, K., Ivanov, P. C., Chen, Z., Carpena, P., Stanley, H. E., 2001. Effect of trends on detrended fluctuation analysis. Phys. Rev. E 64, 011114.

Ibarra, R., Markides, C.N., Matar, O.K., 2014. A review of liquid-liquid flow patterns in horizontal and slightly inclined pipes. Multiphase Sci. Technol., 26, 171-198. 
Ito, S., Hansen, M. E., Heiland, R., Lumsdaine, A., Litke, A. M., Beggs, J. M., 2011. Extending Transfer Entropy improves identification of effective connectivity in a spiking cortical network model. PLoS ONE 6(11), e27431.

Johnsson, F., Zijerveld, R. C., Schouten, J. C., van den Bleek, C. M., Leckner, B., 2006. Characterization of fluidization regimes by time-series analysis of pressure fluctuations. Int. J. Multiphase Flow 26, 663-715.

Kumara, W. A. S., Halvorsen, B. M., Melaaen, M. C., 2009. Pressure drop, flow pattern and local water volume fraction measurements of oil-water flow in pipes. Meas. Sci. Technol. 20,114004 .

Kumara, W. A. S., Halvorsen, B. M., Melaaen, M. C., 2010. Particle image velocimetry for characterizing the flow structure of oil-water flow in horizontal and slightly inclined pipes. Chem. Eng. Sci. 65, 4332-4349.

Kraskov, A., Stoegbauer, H., Grassberger, P., 2004. Estimating mutual information. Phys. Rev. E 69, 066138.

Liang, X. S., Kleeman, R., 2005. Information transfer between dynamical system components. Phys. Rev. Lett. 95(24), 244101.

Liebert, W., Schuster, H. G., 1989. Proper choice of the time delay for the analysis of chaotic time series. Phys. Lett. A 142, 107-111.

Liu, G., Wang, Y. S., Zang, G. J., Zhao, H. T., 2015. Viscous Kelvin-Helmholtz instability analysis of liquid-vapor two-phase stratified flow for condensation in horizontal tubes. Int. J. Heat Mass Tran.84, 592-599.

Llauró, F.X., Llop, M.F., 2006. Characterization and classification of fluidization regimes by non-linear analysis of pressure fluctuations. International Journal of Multiphase Flow 32, 1397-1404.

Llop, M.F., Jand, N., Gallucci, K., Llauró, F.X., 2012. Characterizing gas-solid fluidization by nonlinear tools: Chaotic invariants and dynamic moments. Chemical Engineering Science $71,252-263$.

Lorenz, E. N., 1963. Deterministic nonperiodic flow. J. Atmos. 20, 130-141. 
Lovick, J., Angeli, P., 2004a. Experimental studies on the dual continuous flow pattern in oilwater flows. Int. J. Multiphase Flow 30, 139-157.

Lovick, J. and Angeli, P., 2004b, Droplet size and velocity profiles in liquid-liquid horizontal flows. Chem. Eng. Sci. 59, 3105-3115.

Morgan, R. G., Markides, C. N., Zadrazil, I., Hewitt, G. F., 2013. Characteristics of horizontal liquid-liquid flows in a circular pipe using simultaneous high-speed laser-induced fluorescence and particle velocimetry. Int. J. Multiphase Flow 49, 99-118.

Peng, C. K., Havlin, S., Stanley, H. E., Goldberger, A. L., 1995. Quantification of scaling exponents and crossover phenomena in nonstationary heartbeat time series. Chaos 5(1), 8287.

Picchi, D., Strazza, D., Demori, M., Ferrari, V., Poesio, P., 2015. An experimental investigation and two-fluid model validation for dilute viscous oil in water dispersed pipe flow. Exp. Thermal Fluid Sci. 60, 28-34.

Rodriguez, O. M. H., Oliemans, R. V. A., 2006. Experimental study on oil-water flow in horizontal and slightly inclined pipes. Int. J. Multiphase Flow 32, 323-343.

Russell, T. W. F., Hodgson, G. W., Govier, G. W., 1959. Horizontal pipeline flow of mixtures of oil and water. Can. J. Chem. Eng. 37 (1), 9-17.

Schreiber, T., 2000. Measuring information transfer. Phys. Rev. Lett. 85(2), 461-464.

Shayeganfar, F., 2012. Levels of complexity in turbulent time series for weakly and high Reynolds number. Physica A 391, 3151-3158.

Staniek, M., Lehnertz, K., 2008. Symbolic transfer entropy. Phys. Rev. Lett. 100, 158101.

Torres-Monzón, C. F., 2006. Modeling of Oil-Water Flow in Horizontal and Near Horizontal Pipes. PhD Dissertation, University of Tulsa.

Trallero, J. L., 1995. Oil-water flow patterns in horizontal pipes. Ph.D thesis, The University of Tulsa.

Trallero, J. L., Sarica, C., Brill, J. P., 1997. A study of oil-water flow patterns in horizontal pipes. Spe Production and Facility 12, 165-172. 
van Ommen, J.R., Coppens, M.O., van den Bleek, C.M., Schouten, J.C., 2000. Early warning of agglomeration in fluidized beds by attractor comparison. AIChE Journal 46, 2183-2197.

van Ommen, J.R., Sasic, S., van der Schaaf, J., Gheorghiu, S., Johnsson, F., Coppens, M.O., 2011. Time-series analysis of pressure fluctuations in gas-solid fluidized beds - A review. International Journal of Multiphase Flow 37, 403-428.

Vicente, R., Wibral, M., Lindner, M., Pipa, G., 2011. Transfer entropy-a model-free measure of effective connectivity for the neurosciences. J. Comput. Neurosci. 30, 45-67.

Wang, J., Yu, Z. F., 2012. Symbolic transfer entropy-based premature signal analysis. Chin. Phys. B 21(1), 018702.

Wegmann, A., Rudolf von Rohr, P., 2006. Two phase liquid-liquid flows in pipes of small diameters. Int. J. Multiphase Flow 32, 1017-1028.

Zhai, L. S., Jin, N. D., Gao, Z. K., Zhao, A., Zhu, L., 2014. Cross-correlation velocity measurement of horizontal oil-water two-phase flow by using parallel-wire capacitance probe. Exp. Thermal Fluid Sci. 53, 277-289.

Zhai, L. S., Jin, N. D., Zong, Y. B., Wang, Z. Y., Gu, M., 2012. The development of a conductance method for measuring liquid holdup in horizontal oil-water two-phase flows. Meas. Sci. Technol. 23, 025304.

Zhai, L. S., Jin, N. D., Zong, Y. B., Hao, Q. Y., Gao, Z. K., 2015. Experimental flow pattern map, slippage and time-frequency representation of oil-water two-phase flow in horizontal small diameter pipes. Int. J. Multiphase Flow 76, 168-186.

Zhao, A., Jin, N. D., Ren, Y. Y., Zhu, L., Yang, X., 2016. Multi-scale long-range magnitude and sign correlations in vertical upward oil-gas-water three-phase flow, A Journal of Physical Sciences Zeitschrift für Naturforschung A 71, 33-43.

Zong, Y. B., Jin, N.D., Wang, Z.Y., Gao, Z. K., Wang, C., 2010. Nonlinear dynamic analysis of large diameter inclined oil-water two phase flow pattern. International Journal of Multiphase Flow 36, 166-183. 